\title{
Suboptimal and Optimal MIMO-OFDM Iterative Detection Schemes
}

\author{
Pei Xiao ${ }^{1}$, Zihuai $\operatorname{Lin}^{2}$, Wu Yin ${ }^{3}$, Colin Cowan ${ }^{1}$ \\ ${ }^{1}$ The Institute of Electronics, Communications and Information Technology \\ Queen's University Belfast, BT3 9DT, United Kingdom \\ E-mail: \{pei.xiao, c.cowan\}@qub.ac.uk \\ ${ }^{2}$ School of Electrical and Computer Engineering \\ University of Sydney, 2006 Sydney, Australia \\ E-mail: zihuaidee.usyd.edu.au \\ ${ }^{3}$ ZTE Corporation, Nanshan, Shenzhen, China, 518055 \\ E-mail: yin.wulzte.com.cn
}

\begin{abstract}
A novel iterative detection scheme for MIMOOFDM systems is proposed in this work. We show that the existing detection schemes are sub-optimum and the iterative process can be optimized by utilizing the non-circular property of the residual interference after interference cancellation. Results show that the proposed iterative scheme outperforms the conventional iterative soft interference cancellation (ISIC) and V-BLAST schemes by about 1.7 and $4.0 \mathrm{~dB}$, respectively, in a $4 \times 4$ antennas system over exponentially distributed eleven path channels.
\end{abstract}

\section{INTRODUCTION}

The next generation wireless communication systems call for advanced signal processing techniques to support everincreasing data rates. Multiple-input-multiple-output (MIMO) systems provide an effective means of achieving high data rate transmission without increasing the total transmission power or bandwidth for wireless systems [1]. On the other hand, orthogonal frequency division multiplexing (OFDM) [2], [3] is well suited for broadband applications with high data rate due to its robustness against multipath fading by transforming a frequency selective channel into parallel flat fading channels. The combination of MIMO and OFDM thus provides a powerful measure to build up a reliable broadband wireless communication system. In this context, iterative receivers [4], [5] have been demonstrated to efficiently deal with the coantenna intereference (CAI).

Spatial division multiplexing (SDM) systems achieve high data rate transmission by transmitting multiple substreams simultaneously from multiple transmit antennas. The receiver also has a multiple-antenna architecture to detect spatially multiplexed substreams. For such SDM systems, maximum likelihood (ML) detection has a prohibitive complexity which grows exponentially with the number of antennas and the signal constellation size. The V-BLAST detection technique [6], [7] offers a good tradeoff between performance and complexity. It uses a combination of linear and nonlinear detection techniques: first nulling out the interference from undetected signals, then canceling out the interference using already detected signals. However, the V-BLAST scheme suffers from the error propagation inherent in the decision feedback process. To tackle this problem, we can replicate the CAI components using the log-likelihood ratio (LLR) of the interfering signals and subtract the soft replica from the received compositesignal vector. The performance can be improved by repeating this process in an iterative manner. This is the so called iterative soft interference cancellation (ISIC) technique [8].

The convergence behavior of the iterative ISIC receiver for MIMO-OFDM system is analyzed in [9] using the extrinsic information transfer (EXIT) chart. The joint iterative detection and channel estimation for MIMO-OFDM is treated in [10], where a list sphere decoder (LSD) is employed for MIMO detection and a decision directed SAGE algorithm is used for channel estimation. In [11], the authors presented two iterative space-frequency detection architecutres for the MIMO-OFDM system which incorporates orthogonal space-frequency block coding (OSFBC). The iterative receiver is implemented by either a posteriori probability (APP) space-frequency detector or a soft information aided minimum mean square error (MMSE) combiner. In this paper, we show that the performance of MIMO-OFDM iterative detection can be optimized by utilizing the non-circular nature of the inteference residual after subtracting the CAI using their soft symbol estimates. To this end, a new nulling filter is designed based on a modified error criterion and is applied to remove the residual interference. The remainder of this paper is organized as follows. The system model is described in Section II. The conventional and the improved MIMO-OFDM iterative detection schemes are presented in Section III and IV, respectivey. Numerical results are presented in Section $\mathrm{V}$ to demonstrate the effectiveness of different techniques. Finally, conclusions are drawn in Section VI.

The following notations are used in this paper: $(\cdot)^{\mathcal{T}}$ denotes matrix transpose, $(\cdot)^{\mathcal{H}}$ matrix conjugate transpose, $(\cdot)^{*}$ matrix conjugate, E[·] expectation, and $\mathbf{I}_{N}$ an $N \times N$ identity matrix.

\section{SySTEM MODEL}

We consider a MIMO-OFDM system with $N$ transmit antennas and $M$ receive antennas. First, the binary input bit sequence $\mathbf{b}_{i}(i=1, \ldots, N)$ is mapped to a complex valued data symbol sequence $\mathbf{d}_{i}$. Each block of $N Q$ consecutive data symbols, denoted by $\mathbf{d}=\left[d_{1}, d_{2}, \ldots, d_{N Q}\right]$, is serial-toparallel converted and grouped into $N$ data symbol streams, 
which can be denoted as an $N \times Q$ matrix

$$
\mathbf{D}=\left[\begin{array}{cccc}
d_{1}(1) & d_{1}(2) & \ldots & d_{1}(Q) \\
d_{2}(1) & d_{2}(2) & \ldots & d_{2}(Q) \\
\vdots & \vdots & \ddots & \vdots \\
d_{N}(1) & d_{N}(2) & \ldots & d_{N}(Q)
\end{array}\right]
$$

where each element is denoted as $d_{i}(j) \in \Omega$ where $\Omega$ is a finite set or constellation alphabet, and $Q$ is the number of data subcarriers in one OFDM frame. Each row of the data matrix $\mathbf{D}$ is transmitted by an OFDM module that comprises the inverse Fourier transform (IFFT) and cyclic extension of an OFDM symbol as guard interval or cyclic prefix (CP). The length of $\mathrm{CP}$ should be no shorter than the channel delay spread so as to completely remove inter-symbol interference (ISI) and intercarrier interference (ICI). Finally, the parallel data streams are transmitted by different antenna.

Fig. 1 shows the block diagram of the MIMO-OFDM receiver with $M$ receive antennas. After guard interval removal, the OFDM symbol is transformed into the frequency domain by the fast Fourier transform (FFT). This is followed by $Q$ detectors operating in a parallel fashion, one for each subcarrier. The symbol estimates at the output of the detectors will be demodulated (illustrated by the demapper block in Fig. 1) to obtain an estimate of the transmitted bit sequence $\mathbf{b}_{i}$. Since the same detection mechanism is applied to each detection block, thus only the $k$ th detector will be discussed in the sequel.

After CP removal and FFT operation, the received signal at the $k$ th sub-carrier can be expressed as

$\mathbf{x}(k)=\mathbf{H}(k) \mathbf{d}(k)+\mathbf{u}(k)=\sum_{n=1}^{N} \mathbf{h}_{n}(k) d_{n}(k)+\mathbf{u}(k) \in \mathbb{C}^{M \times 1}$,

where $\mathbf{x}(k)=\left[\begin{array}{llll}x_{1}(k) & x_{2}(k) & \ldots & x_{M}(k)\end{array}\right]^{\mathcal{T}} \quad(k=$ $1,2, \ldots, Q)$ is the received signal vector; $\mathbf{u}(k)=$ $\left[\begin{array}{llll}u_{1}(k) & u_{2}(k) & \ldots & u_{M}(k)\end{array}\right]^{\mathcal{T}}$ denotes the complex additive white Gaussian noise vector with zero mean and covariance matrix $\sigma_{u}^{2} \mathbf{I}_{M}$. The channel matrix $\mathbf{H}(k) \in \mathbb{C}^{M \times N}$ contains the complex channel gains, and can be formed as $\mathbf{H}(k)=$ $\left[\begin{array}{cccc}H_{11}(k) & H_{12}(k) & \ldots & H_{1 N}(k) \\ H_{21}(k) & H_{22}(k) & \ldots & H_{2 N}(k) \\ \vdots & \vdots & \ddots & \vdots \\ H_{M 1}(k) & H_{M 2}(k) & \ldots & H_{M N}(k)\end{array}\right]$, where $H_{p q}(k)$ is the frequency response of the channel between the $p$ th receive antenna and the $q$ th transmit antenna at the $k$ th sub-carrier. The transmitted symbol vector $\mathbf{d}(k)=\left[\begin{array}{llll}d_{1}(k) & \ldots & d_{N}(k)\end{array}\right]^{\mathcal{T}}$ is the $k$ th column of the matrix $\mathbf{D}$ expressed in (1). The data symbols are assumed to be uncorrelated and to have zero mean and unit energy, i.e., $\mathrm{E}\left[\mathbf{d}(k) \mathbf{d}^{\mathcal{H}}(k)\right]=\mathbf{I}_{N}$. The vector $\mathbf{h}_{n}(k)$ is the $n$th column of $\mathbf{H}(k)$. The index $k$ will be omitted in the sequel to simplify notations.

Now let us see how the symbol $d_{n}, n=1, \ldots, N$ can be decoded. After performing interference cancellation, the interference canceled version of the received vector is given as

$$
\mathbf{x}_{n}=\mathbf{x}-\mathbf{H} \overline{\mathbf{d}}_{n}=\mathbf{H}\left[\mathbf{d}-\overline{\mathbf{d}}_{n}\right]+\mathbf{u} \in \mathbb{C}^{N \times 1},
$$

where

$$
\overline{\mathbf{d}}_{n}=\left[\begin{array}{lllllll}
\bar{d}_{1} & \ldots & \bar{d}_{n-1} & 0 & \bar{d}_{n+1} & \ldots & \bar{d}_{N}
\end{array}\right]^{\mathcal{T}},
$$

contains the soft estimate of the interference symbols from the previous iteration. Note that (3) represents a decisiondirected ISIC scheme, where the symbol estimates from the previous iteration are used for the detection procedure at the current iteration. For simplicity, the iteration index is omitted, whenever no ambiguity arises.

\section{Conventional Iterative Scheme}

In order to further suppress the residual interference in $\mathbf{x}_{n}$, a nulling vector $\mathbf{m}_{n}$ is applied to $\mathbf{x}_{n}$, to obtain $z_{n}=$ $\mathbf{m}_{n}^{\mathcal{H}} \mathbf{x}_{n}$, where $\mathbf{m}_{n} \in \mathbb{C}^{M \times 1}$ is chosen by minimizing $e_{n}=$ $\mathrm{E}\left\{\left|\mathbf{m}_{n}^{\mathcal{H}} \mathbf{x}_{n}-d_{n}\right|^{2}\right\}$ under the MMSE criterion. It can be derived as [5]

$$
\mathbf{m}_{n}=\left[\mathbf{H} \boldsymbol{\Lambda}_{n} \mathbf{H}^{\mathcal{H}}+\sigma_{u}^{2} \mathbf{I}_{M}\right]^{-1} \mathbf{h}_{n}
$$

The matrix $\boldsymbol{\Lambda}_{n} \in \mathbb{R}^{N \times 1}$ is formed as

$$
\begin{aligned}
& \boldsymbol{\Lambda}_{n}=\operatorname{diag}\left\{\left[1-\left|\bar{d}_{1}\right|^{2} \quad \ldots \quad 1-\left|\bar{d}_{n-1}\right|^{2}\right.\right. \\
& \left.\left.11-\left|\bar{d}_{n+1}\right|^{2} \quad \ldots \quad 1-\left|\bar{d}_{N}\right|^{2}\right]\right\} ;
\end{aligned}
$$

In what follows, we use QPSK systems as an example to demonstrate how the vector $\overline{\mathbf{d}}_{n}$ in (4) and the matrix $\boldsymbol{\Lambda}_{n}$ in (6) can be derived in order to carry out the iterative process.

The filter output can be expressed as $z_{n}=\mathbf{m}_{n}^{\mathcal{H}} \mathbf{x}_{n}=\mu_{n} d_{n}+$ $\xi_{n}$, where the combined noise and residual interference $\xi_{n}$ can be approximated as a Gaussian random variable [12], i.e., $\xi_{n} \sim \mathcal{C N}\left(0, N_{\xi}\right)$. The parameters $\mu_{n}, N_{\xi}$ can be determined as

$$
\begin{aligned}
\mu_{n} & =\mathrm{E}\left\{z_{n} d_{n}^{*}\right\}=\mathbf{m}_{n}^{\mathcal{H}} \mathrm{E}\left[\mathbf{x}_{n} d_{n}^{*}\right]=\mathbf{m}_{n}^{\mathcal{H}} \mathbf{C}_{\mathbf{x} d}=\mathbf{m}_{n}^{\mathcal{H}} \mathbf{h}_{n} \\
N_{\xi} & =\mathrm{E}\left[\left|\xi_{n}\right|^{2}\right]=\mathrm{E}\left[\left|z_{n}-\mu_{n} d_{n}\right|^{2}\right] \\
& =\mathrm{E}\left\{\left|z_{n}\right|^{2}\right\}-\left|\mu_{n}\right|^{2}=\mu_{n}\left(1-\mu_{n}\right) .
\end{aligned}
$$

After computing the values of $\mu_{n}$ and $N_{\xi}$, the conditional probability density function (PDF) of the filter output can be obtained as

$$
f\left(z_{n} \mid d_{n}=s_{m}\right)=\frac{1}{\pi N_{\xi}} \exp \left(-\frac{\left|z_{n}-\mu_{n} s_{m}\right|^{2}}{N_{\xi}}\right),
$$

In QPSK systems, each symbol $d_{n}$ corresponds to two information bits, denoted as $b_{n}^{0}$ and $b_{n}^{1}$. Denoting the loglikelihood ratio (LLR) value of $d_{n}$ as $\lambda\left(d_{n}\right)=\lambda\left(b_{n}^{0}\right)+j \lambda\left(b_{n}^{1}\right)$, the soft estimate of $d_{n}$ is computed according to its LLR value as

$$
\bar{d}_{n}=\tanh \left[\lambda\left(b_{n}^{0}\right) / 2\right] / \sqrt{2}+j \tanh \left[\lambda\left(b_{n}^{1}\right) / 2\right] / \sqrt{2} .
$$

The LLR for the first information bit $b_{n}^{0}$ can be computed as

$$
\begin{aligned}
\lambda\left(b_{n}^{0}\right) & =\ln \frac{f\left(z_{n} \mid b_{n}^{0}=1\right)}{f\left(z_{n} \mid b_{n}^{0}=0\right)}=\ln \frac{f\left(z_{n} \mid d_{n}=s_{3}\right)+f\left(z_{n} \mid d_{n}=s_{4}\right)}{f\left(z_{n} \mid d_{n}=s_{1}\right)+f\left(z_{n} \mid d_{n}=s_{2}\right)} \\
& \approx \ln \frac{\exp \left(-\left|z_{n}-\mu_{n} d_{n}^{+}\right|^{2} / N_{\xi}\right)}{\exp \left(-\left|z_{n}-\mu_{n} d_{n}^{-}\right|^{2} / N_{\xi}\right)} \\
& =\frac{1}{N_{\xi}}\left\{\left|z_{n}-\mu_{n} d_{n}^{-}\right|^{2}-\left|z_{n}-\mu_{n} d_{n}^{+}\right|^{2}\right\} \\
& =\frac{2}{1-\mu_{n}} \operatorname{Re}\left\{d_{n}^{+*} z_{n}-d_{n}^{-*} z_{n}\right\}
\end{aligned}
$$




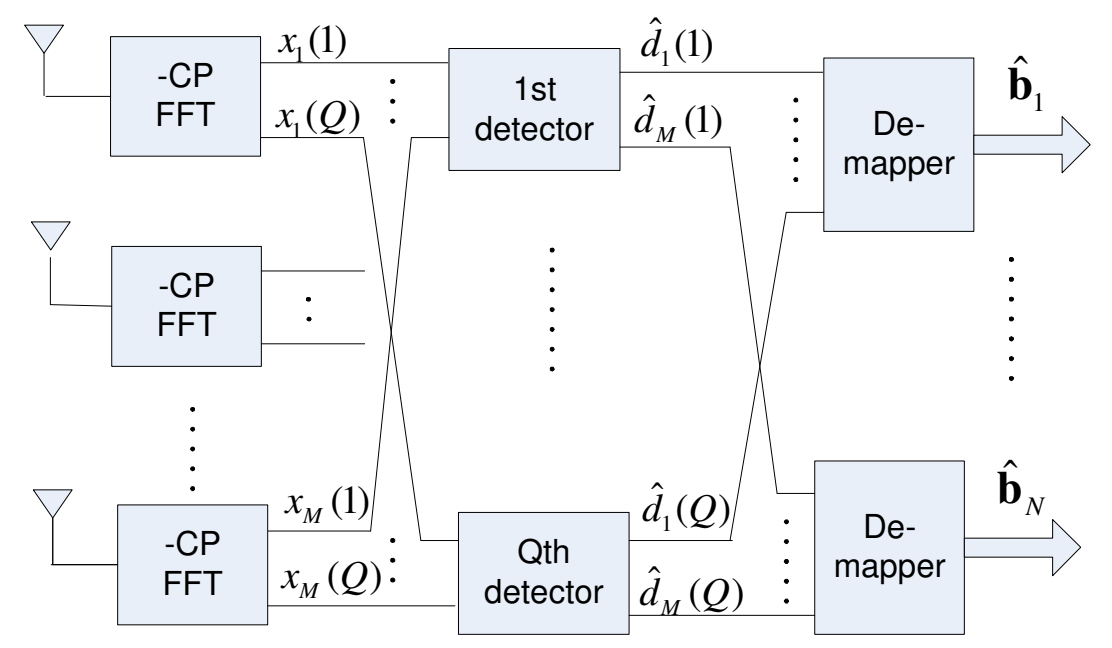

Fig. 1. Block diagram of the MIMO-OFDM receiver.

where $d_{n}^{+}$denotes the QPSK symbol corresponding to $\max \left\{f\left(z_{n} \mid d_{n}=s_{3}\right), f\left(z_{n} \mid d_{n}=s_{4}\right)\right\}$, and $d_{n}^{-}$denotes the QPSK symbol corresponding to $\max \left\{f\left(z_{n} \mid d_{n}=\right.\right.$ $\left.\left.s_{1}\right), f\left(z_{n} \mid d_{n}=s_{2}\right)\right\}$ since the real part of the symbols $s_{3}, s_{4}$ corresponds to 1 , and the real part of the symbols $s_{1}, s_{2}$ corresponds to 0 .

The LLR value for the second information bit can be obtained in a similar manner as

$$
\begin{aligned}
\lambda\left(b_{n}^{1}\right) & =\ln \frac{f\left(z_{n} \mid d_{n}=s_{2}\right)+f\left(z_{n} \mid d_{n}=s_{4}\right)}{f\left(z_{n} \mid d_{n}=s_{1}\right)+f\left(z_{n} \mid d_{n}=s_{3}\right)} \\
& \approx \frac{2}{1-\mu_{n}} \operatorname{Re}\left\{\tilde{d}_{n}^{+*} z_{n}-\tilde{d}_{n}^{-*} z_{n}\right\}
\end{aligned}
$$

where $\tilde{d}_{n}^{+}$denotes the QPSK symbol corresponding to $\max \left\{f\left(z_{n} \mid d_{n}=s_{2}\right), f\left(z_{n} \mid d_{n}=s_{4}\right)\right\}$, and $\tilde{d}_{n}^{-}$denotes the QPSK symbol corresponding to $\max \left\{f\left(z_{n} \mid d_{n}=\right.\right.$ $\left.\left.s_{1}\right), f\left(z_{n} \mid d_{n}=s_{3}\right)\right\}$ since the imaginary part of the symbols $s_{2}, s_{4}$ corresponds to 1 , and the imaginary part of the symbols $s_{1}, s_{3}$ corresponds to 0 .

\section{Proposed Iterative Scheme}

Let us re-define the detection error as $\epsilon_{n}=z_{n}-d_{n}=$ $\boldsymbol{w}_{n}^{\mathcal{H}} \mathbf{y}_{n}-d_{n}$, where $\mathbf{y}_{n}=\left[\begin{array}{ll}\mathbf{x}_{n} & \mathbf{x}_{n}^{*}\end{array}\right]^{\mathcal{T}}$. According to the orthogonality principle [13], the mean-square value of the estimation error $\epsilon_{n}$ is only minimal, if it is orthogonal to the observation vector $\mathbf{y}_{n}$, i.e.,

$$
\mathrm{E}\left[\mathbf{y}_{n} \epsilon_{n}^{*}\right]=\mathrm{E}\left[\mathbf{y}_{n}\left(\boldsymbol{w}_{n}^{\mathcal{H}} \mathbf{y}_{n}-d_{n}\right)^{\mathcal{H}}\right]=\mathbf{0},
$$

leading to the solution $\boldsymbol{w}_{n}=\mathbf{C}_{\mathbf{y y}}^{-1} \mathbf{C}_{\mathbf{y} d}$, where

$$
\begin{aligned}
& \mathbf{C}_{\mathbf{y y}}=\mathrm{E}\left\{\mathbf{y}_{n} \mathbf{y}_{n}^{\mathcal{H}}\right\}=\mathrm{E}\left\{\left[\begin{array}{c}
\mathbf{x}_{n} \\
\mathbf{x}_{n}^{*}
\end{array}\right]\left[\begin{array}{ll}
\mathbf{x}_{n}^{\mathcal{H}} & \mathbf{x}_{n}^{\mathcal{T}}
\end{array}\right]\right\}=\left[\begin{array}{ll}
\mathbf{C}_{\mathbf{x x}} & \tilde{\mathbf{C}}_{\mathbf{x x}} \\
\tilde{\mathbf{C}}_{\mathbf{x x}}^{*} & \mathbf{C}_{\mathbf{x x}}^{*}
\end{array}\right], \\
& \mathbf{C}_{\mathbf{y} d}=\mathrm{E}\left\{\mathbf{y}_{n} d_{n}^{*}\right\}=\mathrm{E}\left\{\left[\begin{array}{l}
\mathbf{x}_{n} \\
\mathbf{x}_{n}^{*}
\end{array}\right] d_{n}^{*}\right\}=\left[\begin{array}{c}
\mathbf{C}_{\mathbf{x} d} \\
\tilde{\mathbf{C}}_{\mathbf{x} d}^{*}
\end{array}\right]=\left[\begin{array}{c}
\mathbf{h}_{n} \\
\mathbf{0}
\end{array}\right]
\end{aligned}
$$

The matrix $\tilde{\mathbf{C}}_{\mathbf{x} d}^{*}=\mathbf{0}$ since $\mathrm{E}\left[\left(d_{i}-\bar{d}_{i}\right)^{*} d_{n}^{*}\right]=0$ if $i \neq n$; and $\mathrm{E}\left[d_{n}^{*} d_{n}^{*}\right]=0$ for complex signal constellations. The autocorrelation matrix $\mathbf{C}_{\mathbf{x x}}$ and the pseudo-autocorrelation matrix $\tilde{\mathbf{C}}_{\mathbf{x x}}$ can be computed as $\mathbf{C}_{\mathbf{x x}}=\mathbf{H} \boldsymbol{\Lambda}_{n} \mathbf{H}^{\mathcal{H}}+\sigma_{u}^{2} \mathbf{I}$ and $\tilde{\mathbf{C}}_{\mathbf{x x}}=\mathbf{H} \tilde{\boldsymbol{\Lambda}}_{n} \mathbf{H}^{\mathcal{T}}$, respectively. The matrix $\tilde{\boldsymbol{\Lambda}}_{n}$ is defined as

$$
\begin{aligned}
\tilde{\boldsymbol{\Lambda}}_{n} & =\operatorname{E}\left\{\left[\mathbf{d}_{n}-\overline{\mathbf{d}}_{n}\right]\left[\mathbf{d}_{n}-\overline{\mathbf{d}}_{n}\right]^{\mathcal{T}}\right\} \\
& =\operatorname{diag}\left\{\begin{array}{lllllll}
\Lambda_{1} & \ldots & \Lambda_{n-1} & 0 & \Lambda_{n+1} & \ldots & \left.\Lambda_{N}\right\}
\end{array}\right.
\end{aligned}
$$

Denoting the complex symbol $d_{i}=d_{i, I}+j d_{i, Q}$, and $\bar{d}_{i}=$ $\bar{d}_{i, I}+j \bar{d}_{i, Q}$, where $\bar{d}_{i}=\mathrm{E}\left[d_{i}\right]$, the $p$ th diagonal element of $\tilde{\Lambda}_{n}$ is calculated as

$$
\begin{aligned}
\Lambda_{p}= & \mathrm{E}\left[\left(d_{i}-\bar{d}_{i}\right)^{2}\right]=\mathrm{E}\left[d_{i}^{2}\right]-\left(\bar{d}_{i}\right)^{2} \\
= & \mathrm{E}\left[d_{i, I}^{2}+2 j d_{i, I} d_{i, Q}-d_{i, Q}^{2}\right]-\left(\bar{d}_{i, I}\right)^{2} \\
& -2 j \bar{d}_{i, I} \bar{d}_{i, Q}+\left(\bar{d}_{i, Q}\right)^{2}=\left(\bar{d}_{i, Q}\right)^{2}-\left(\bar{d}_{i, I}\right)^{2} .
\end{aligned}
$$

Based on the above analysis, we can form the new nulling filter as

$\boldsymbol{w}_{n}=\mathbf{C}_{\mathbf{y y}}^{-1} \mathbf{C}_{\mathbf{y} d}=\left[\begin{array}{cc}\mathbf{H} \boldsymbol{\Lambda}_{n} \mathbf{H}^{\mathcal{H}}+\sigma_{u}^{2} \mathbf{I} & \mathbf{H} \tilde{\boldsymbol{\Lambda}}_{n} \mathbf{H}^{\mathcal{T}} \\ \mathbf{H}^{*} \tilde{\boldsymbol{\Lambda}}_{n}^{*} \mathbf{H}^{\mathcal{H}} & \mathbf{H}^{*} \boldsymbol{\Lambda}_{n} \mathbf{H}^{\mathcal{T}}+\sigma_{u}^{2} \mathbf{I}\end{array}\right]^{-1}\left[\begin{array}{c}\mathbf{h}_{n} \\ \mathbf{0}\end{array}\right]$.

Note that the conventional filter coefficient vector $\mathbf{m}_{n}$ in (5) is calculated using only the autocorrelation matrix $\mathbf{C}_{\mathbf{x x}}=$ $\mathrm{E}\left[\mathbf{x}_{n} \mathbf{x}_{n}^{\mathcal{H}}\right]=\mathbf{H} \boldsymbol{\Lambda}_{n} \mathbf{H}^{\mathcal{H}}+\sigma_{u}^{2} \mathbf{I}$, i.e., $\mathbf{m}_{n}=\mathbf{C}_{\mathbf{x x}}^{-1} \mathbf{C}_{\mathbf{x} d}$. The pseudo-autocorrelation matrix $\tilde{\mathbf{C}}_{\mathbf{x x}}$ is implicitly assumed to be zero, leading to sub-optimum solutions.

The nulling filter output can be expressed as $z_{n}=\mu_{n} d_{n}+$ $\nu_{n} d_{n}^{*}+\eta_{n}$, where the combined noise and residual interference $\eta_{n}$ can be approximated as a Gaussian random variable. Next, we derive the LLR values for $b_{n}^{0}$ and $b_{n}^{1}$ for QPSK systems based on the assumption that the interference-plus-noise term $\eta_{n}$ at the output of the nulling filter is also a non-circular random process. The parameters $\mu_{n}, \nu_{n}, N_{\eta}$ can be computed 
as

$$
\begin{aligned}
\mu_{n} & =\mathrm{E}\left\{z_{n} d_{n}^{*}\right\}=\boldsymbol{w}_{n}^{\mathcal{H}} \mathrm{E}\left[\mathbf{y}_{n} d_{n}^{*}\right]=\boldsymbol{w}_{n}^{\mathcal{H}} \mathbf{C}_{\mathbf{y} d}=\boldsymbol{w}_{n}^{\mathcal{H}}\left[\begin{array}{c}
\mathbf{v}_{n} \\
\mathbf{0}
\end{array}\right] \\
\nu_{n} & =\mathrm{E}\left\{z_{n} d_{n}\right\}=\boldsymbol{w}_{n}^{\mathcal{H}} \mathrm{E}\left[\mathbf{y}_{n} d_{n}\right]=\boldsymbol{w}_{n}^{\mathcal{H}} \tilde{\mathbf{C}}_{\mathbf{y} d}=\boldsymbol{w}_{n}^{\mathcal{H}}\left[\begin{array}{c}
\mathbf{0} \\
\mathbf{v}_{n}^{*}
\end{array}\right] \\
N_{\eta} & =\mathrm{E}\left[\left|\eta_{n}\right|^{2}\right]=\mathrm{E}\left[\left|z_{n}-\mu_{n} d_{n}-\nu_{n} d_{n}^{*}\right|^{2}\right] \\
& =\mathrm{E}\left\{\left|z_{n}\right|^{2}\right\}-\left|\mu_{n}\right|^{2}-\left|\nu_{n}\right|^{2}=\mu_{n}^{*}-\left|\mu_{n}\right|^{2}-\left|\nu_{n}\right|^{2} .
\end{aligned}
$$

The above equation holds since $z_{n}=\boldsymbol{w}_{n}^{\mathcal{H}} \mathbf{y}_{n}$ and $\boldsymbol{w}_{n}=$ $\mathbf{C}_{\mathbf{y y}}^{-1} \mathbf{C}_{\mathbf{y} d}$. Therefore,

$$
\begin{aligned}
\mathrm{E}\left\{\left|z_{n}\right|^{2}\right\} & =\mathrm{E}\left\{\boldsymbol{w}_{n}^{\mathcal{H}} \mathbf{y}_{n} \mathbf{y}_{n}^{\mathcal{H}} \boldsymbol{w}_{n}\right\}=\boldsymbol{w}_{n}^{\mathcal{H}} \mathbf{C}_{\mathbf{y y}} \boldsymbol{w}_{n} \\
& =\mathbf{C}_{\mathbf{y} d}^{\mathcal{H}} \mathbf{C}_{\mathbf{y y}}^{-1} \mathbf{C}_{\mathbf{y y}} \boldsymbol{w}_{n}=\mathbf{C}_{\mathbf{y} d}^{\mathcal{H}} \boldsymbol{w}_{n}=\mu_{n}^{*} .
\end{aligned}
$$

In the derivation of the proposed scheme, we take into account the non-circular nature of $\eta_{n}$, and utilize the fact that $\tilde{N}_{\eta}=\mathrm{E}\left[\eta_{n}^{2}\right] \neq 0$, which can be computed as

$$
\begin{aligned}
\tilde{N}_{\eta} & =\mathrm{E}\left[\eta^{2}\right]=\mathrm{E}\left[\left(z_{n}-\mu_{n} d_{n}-\nu_{n} d_{n}^{*}\right)^{2}\right] \\
& =\mathrm{E}\left\{z_{n}^{2}\right\}-2 \mu_{n} \nu_{n}=\mathrm{E}\left\{\boldsymbol{w}_{n}^{\mathcal{H}} \mathbf{y}_{n} \mathbf{y}_{n}^{\mathcal{T}} \boldsymbol{w}_{n}^{*}\right\}-2 \mu_{n} \nu_{n} \\
& =\boldsymbol{w}_{n}^{\mathcal{H}} \tilde{\mathbf{C}}_{\mathbf{y y}} \boldsymbol{w}_{n}^{*}-2 \mu_{n} \nu_{n} .
\end{aligned}
$$

The above equation holds since $\boldsymbol{w}_{n}^{\mathcal{H}} \mathbf{y}=\mathbf{y}_{n}^{\mathcal{T}} \boldsymbol{w}_{n}^{*}$. Therefore $\mathrm{E}\left\{z_{n}^{2}\right\}=\mathrm{E}\left\{\boldsymbol{w}_{n}^{\mathcal{H}} \mathbf{y}_{n} \mathbf{y}_{n}^{\mathcal{T}} \boldsymbol{w}_{n}^{*}\right\}=\boldsymbol{w}_{n}^{\mathcal{H}} \mathrm{E}\left\{\mathbf{y}_{n} \mathbf{y}_{n}^{\mathcal{T}}\right\} \boldsymbol{w}_{n}^{*}=\boldsymbol{w}_{n}^{\mathcal{H}} \tilde{\mathbf{C}}_{\mathbf{y y}} \boldsymbol{w}_{n}^{*}$,

where

$$
\begin{aligned}
\tilde{\mathbf{C}}_{\mathbf{y y}} & =\mathrm{E}\left\{\mathbf{y}_{n} \mathbf{y}_{n}^{\mathcal{T}}\right\}=\mathrm{E}\left\{\left[\begin{array}{c}
\mathbf{x}_{n} \\
\mathbf{x}_{n}^{*}
\end{array}\right]\left[\begin{array}{ll}
\mathbf{x}_{n}^{\mathcal{T}} & \mathbf{x}_{n}^{\mathcal{H}}
\end{array}\right]\right\}=\left[\begin{array}{ll}
\tilde{\mathbf{C}}_{\mathbf{x x}} & \mathbf{C}_{\mathbf{x x}} \\
\mathbf{C}_{\mathbf{x x}}^{*} & \tilde{\mathbf{C}}_{\mathbf{x x}}^{*}
\end{array}\right] \\
& =\left[\begin{array}{cc}
\mathbf{H} \tilde{\mathbf{\Lambda}}_{n} \mathbf{H}^{\mathcal{T}} & \mathbf{H} \mathbf{\Lambda}_{n} \mathbf{H}^{\mathcal{H}}+\sigma_{u}^{2} \mathbf{I} \\
\mathbf{H}^{*} \boldsymbol{\Lambda}_{n} \mathbf{H}^{\mathcal{T}}+\sigma_{u}^{2} \mathbf{I} & \mathbf{H}^{*} \tilde{\mathbf{\Lambda}}_{n}^{*} \mathbf{H}^{\mathcal{H}}
\end{array}\right] ;
\end{aligned}
$$

Let us denote $z_{n}=z_{n, I}+j z_{n, Q}, d_{n}=d_{n, I}+j d_{n, Q}$, and $\eta_{n}=\eta_{n, I}+j \eta_{n, Q}$. The filter output $z_{n}=\mu_{n} d_{n}+\nu_{n} d_{n}^{*}+\eta_{n}$ can be reformed as $\underbrace{\left[\begin{array}{c}z_{n, I} \\ z_{n, Q}\end{array}\right]}_{\mathbf{z}_{n}}=\underbrace{\left[\begin{array}{c}\left(\mu_{n}+\nu_{n}\right) d_{n, I} \\ \left(\mu_{n}-\nu_{n}\right) d_{n, Q}\end{array}\right]}_{\mathbf{d}_{n}}+\underbrace{\left[\begin{array}{c}\eta_{n, I} \\ \eta_{n, Q}\end{array}\right]}_{\boldsymbol{\eta}_{n}}$.

Since the probability distribution of a complex random variable or vector is a joint distribution of its real and imaginary part, we have

$$
\begin{aligned}
& f\left(z_{n} \mid d_{n}\right)=f\left(\mathbf{z}_{n} \mid \mathbf{d}_{n}\right) \\
& =\frac{1}{2 \pi \sqrt{\operatorname{det} \boldsymbol{\Sigma}_{n}}} \exp \left(-\frac{1}{2}\left(\mathbf{z}_{n}-\mathbf{d}_{n}\right)^{\mathcal{H}} \boldsymbol{\Sigma}_{n}^{-1}\left(\mathbf{z}_{n}-\mathbf{d}_{n}\right)\right),
\end{aligned}
$$

where the covariance matrix of the Gaussian noise is $\boldsymbol{\Sigma}_{n}=$ $\mathrm{E}\left[\boldsymbol{\eta}_{n} \boldsymbol{\eta}_{n}^{\mathcal{H}}\right]$. Define the mapping matrix as $\mathbf{J}=\frac{1}{\sqrt{2}}\left[\begin{array}{cc}1 & j \\ 1 & -j\end{array}\right]$, which is an unitary matrix since $\mathbf{J} \mathbf{J}^{\mathcal{H}}=\mathbf{J}^{\mathcal{H}} \mathbf{J}=\mathbf{I}$, and $\mathbf{J}^{-1}=$ $\mathbf{J}^{\mathcal{H}}$. We have

$$
\begin{aligned}
\mathbf{J} \boldsymbol{\Sigma}_{n} \mathbf{J}^{\mathcal{H}} & =\mathbf{J} \mathrm{E}\left[\boldsymbol{\eta}_{n} \boldsymbol{\eta}_{n}^{\mathcal{H}}\right] \mathbf{J}^{\mathcal{H}}=\mathrm{E}\left[\left(\mathbf{J} \boldsymbol{\eta}_{n}\right)\left(\mathbf{J} \boldsymbol{\eta}_{n}\right)^{\mathcal{H}}\right] \\
& =\frac{1}{2} \mathrm{E}\left[\boldsymbol{\epsilon}_{n} \boldsymbol{\epsilon}_{n}^{\mathcal{H}}\right]=\frac{1}{2} \boldsymbol{\Phi}_{n},
\end{aligned}
$$

where $\boldsymbol{\epsilon}_{n}=\left[\begin{array}{c}\eta_{n} \\ \eta_{n}^{*}\end{array}\right]$, and

$$
\begin{aligned}
\boldsymbol{\Phi}_{n} & =\mathrm{E}\left[\boldsymbol{\epsilon}_{n} \boldsymbol{\epsilon}_{n}^{\mathcal{H}}\right]=\mathrm{E}\left\{\left[\begin{array}{c}
\eta_{n} \\
\eta_{n}^{*}
\end{array}\right]\left[\begin{array}{ll}
\eta_{n}^{*} & \eta_{n}
\end{array}\right]\right\} \\
& =\mathrm{E}\left\{\left[\begin{array}{ll}
\eta_{n} \eta_{n}^{*} & \eta_{n} \eta_{n} \\
\eta_{n}^{*} \eta_{n}^{*} & \eta_{n}^{*} \eta_{n}
\end{array}\right]\right\}=\left[\begin{array}{cc}
N_{\eta} & \tilde{N}_{\eta} \\
\tilde{N}_{\eta}^{*} & N_{\eta}
\end{array}\right]
\end{aligned}
$$

From (12), we have $\boldsymbol{\Sigma}_{n}=\frac{1}{2} \mathbf{J}^{\mathcal{H}} \boldsymbol{\Phi}_{n} \mathbf{J}$, and $\boldsymbol{\Sigma}_{n}^{-1}=$ $2 \mathbf{J}^{\mathcal{H}} \boldsymbol{\Phi}_{n}^{-1} \mathbf{J}$. The PDF in (11) can thus be reformed as $f\left(z_{n} \mid d_{n}\right)=\frac{1}{2 \pi \sqrt{\operatorname{det} \boldsymbol{\Sigma}_{n}}} \exp \left[-\left(\mathbf{z}_{n}-\mathbf{d}_{n}\right)^{\mathcal{H}} \mathbf{J}^{\mathcal{H}} \boldsymbol{\Phi}_{n}^{-1} \mathbf{J}\left(\mathbf{z}_{n}-\mathbf{d}_{n}\right)\right]$.

The LLR value of $b_{n}^{0}$ can thus be computed as

$$
\begin{aligned}
\lambda\left(b_{n}^{0}\right)= & \ln \frac{f\left(z_{n} \mid b_{n}^{0}=1\right)}{f\left(z_{n} \mid b_{n}^{0}=0\right)}=\ln \frac{f\left(z_{n} \mid d_{n, I}=+1 / \sqrt{2}\right)}{f\left(z_{n} \mid d_{n, I}=-1 / \sqrt{2}\right)} \\
\approx & \left.\ln \frac{\exp \left[-\left(\mathbf{z}_{n}-\mathbf{d}_{+}\right)^{\mathcal{H}} \mathbf{J}^{\mathcal{H}} \boldsymbol{\Phi}_{n}^{-1} \mathbf{J}\left(\mathbf{z}_{n}-\mathbf{d}_{+}\right)\right]}{\exp \left[-\left(\mathbf{z}_{n}-\mathbf{d}_{-}\right)^{\mathcal{H}} \mathbf{J}\right.} \mathbf{J}_{n}^{-1} \mathbf{J}\left(\mathbf{z}_{n}-\mathbf{d}_{-}\right)\right] \\
= & \left(\mathbf{z}_{n}-\mathbf{d}_{-}\right)^{\mathcal{H}} \mathbf{J}^{\mathcal{H}} \mathbf{\Phi}_{n}^{-1} \mathbf{J}\left(\mathbf{z}_{n}-\mathbf{d}_{-}\right) \\
& -\left(\mathbf{z}_{n}-\mathbf{d}_{+}\right)^{\mathcal{H}} \mathbf{J}^{\mathcal{H}} \boldsymbol{\Phi}_{n}^{-1} \mathbf{J}\left(\mathbf{z}_{n}-\mathbf{d}_{+}\right),
\end{aligned}
$$

where $\mathbf{d}_{+}$denotes the vector $\mathbf{d}_{n}$ corresponding to

$$
\begin{gathered}
\max \left\{f\left(\mathbf{z}_{n} \mid d_{n, I}=+1 / \sqrt{2}, d_{n, Q}=+1 / \sqrt{2}\right),\right. \\
\left.f\left(\mathbf{z}_{n} \mid d_{n, I}=+1 / \sqrt{2}, d_{n, Q}=-1 / \sqrt{2}\right)\right\} ;
\end{gathered}
$$

and $\mathbf{d}_{-}$denotes the vector $\mathbf{d}_{n}$ corresponding to

$$
\begin{gathered}
\max \left\{f\left(\mathbf{z}_{n} \mid d_{n, I}=-1 / \sqrt{2}, d_{n, Q}=+1 / \sqrt{2}\right),\right. \\
\left.f\left(\mathbf{z}_{n} \mid d_{n, I}=-1 / \sqrt{2}, d_{n, Q}=-1 / \sqrt{2}\right)\right\}
\end{gathered}
$$

Similarly

$$
\begin{aligned}
\lambda\left(b_{n}^{1}\right)= & \ln \frac{f\left(z_{n} \mid b_{n}^{1}=1\right)}{f\left(z_{n} \mid b_{n}^{1}=0\right)}=\ln \frac{f\left(z_{n} \mid d_{n, Q}=+1 / \sqrt{2}\right)}{f\left(z_{n} \mid d_{n, I}=-1 / \sqrt{2}\right)} \\
\approx & \left(\mathbf{z}_{n}-\tilde{\mathbf{d}}_{-}\right)^{\mathcal{H}} \mathbf{J}^{\mathcal{H}} \boldsymbol{\Phi}_{n}^{-1} \mathbf{J}\left(\mathbf{z}_{n}-\tilde{\mathbf{d}}_{-}\right) \\
& -\left(\mathbf{z}_{n}-\tilde{\mathbf{d}}_{+}\right)^{\mathcal{H}} \mathbf{J}^{\mathcal{H}} \boldsymbol{\Phi}_{n}^{-1} \mathbf{J}\left(\mathbf{z}_{n}-\tilde{\mathbf{d}}_{+}\right),
\end{aligned}
$$

where $\tilde{\mathbf{d}}_{+}$denotes the vector $\mathbf{d}_{n}$ corresponding to

$$
\begin{gathered}
\max \left\{f\left(\mathbf{z}_{n} \mid d_{n, I}=+1 / \sqrt{2}, d_{n, Q}=+1 / \sqrt{2}\right),\right. \\
\left.f\left(\mathbf{z}_{n} \mid d_{n, I}=-1 / \sqrt{2}, d_{n, Q}=+1 / \sqrt{2}\right)\right\} ;
\end{gathered}
$$

and $\tilde{\mathbf{d}}_{-}$denotes the vector $\mathbf{d}_{n}$ corresponding to

$$
\begin{gathered}
\max \left\{f\left(\mathbf{z}_{n} \mid d_{n, I}=+1 / \sqrt{2}, d_{n, Q}=-1 / \sqrt{2}\right),\right. \\
\left.f\left(\mathbf{z}_{n} \mid d_{n, I}=-1 / \sqrt{2}, d_{n, Q}=-1 / \sqrt{2}\right)\right\}
\end{gathered}
$$

Then we use Equ. (8) to convert LLRs to soft symbol estimate $\bar{d}_{n}$, which is needed for the interference cancellation at the next iteration.

\section{Numerical Results}

The performance of the uncoded MIMO-OFDM system under study is evaluated through computer simulations. Both transmitter and receiver have 4 antennas, i.e., $N=M=$ 4. A 256-point IFFT/FFT is employed for OFDM modulation/demodulation. A tapped-delay-line channel model is used, it has 11 taps with an exponentially decaying power delay profile, following the channel model adopted by the IEEE 802.11 standard [14]. The total channel gain is normalized to unity. The length of CP is set to be $L_{c p}=16$.

Fig. 2 shows the performance comparison between the different algorithms. The curves for the conventional ISIC and the proposed scheme are plotted at the 3rd iteration when the convergence is reached. For the V-BLAST detector [7], its nulling vectors are also designed under the MMSE 


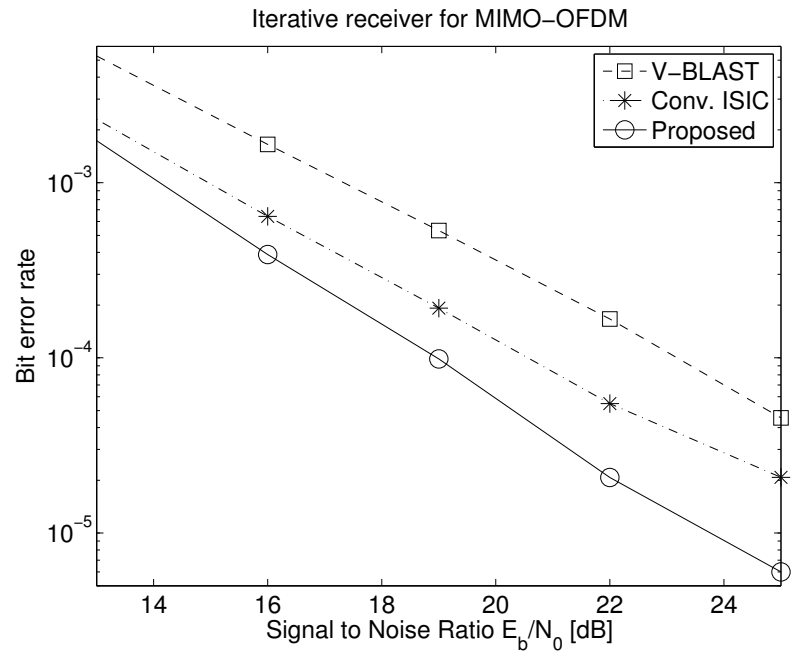

Fig. 2. Performance comparison of different schemes.

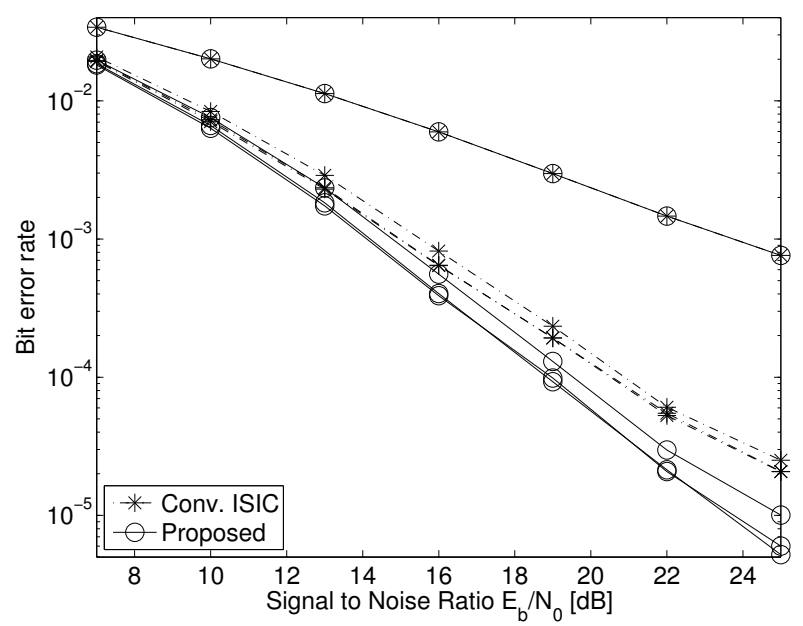

Fig. 3. Convergence behavior of the iterative schemes. The topmost curve represents the performance of different schemes at the 1st iteration; the bottom curve represents the performance of different schemes at the 4th iteration.

criterion. By comparison, the proposed detector outperforms the conventional ISIC detector and the V-BLAST detector by 1.7 and $4.0 \mathrm{~dB}$, respectively, at bit error rate of $10^{-4}$. The V-BLAST detector suffers from the error propagation problem, inaccurate replica miss-cancels the interference and errors would be propagated into the following interference cancellation stages. The conventional ISIC scheme alleviates this problem by using soft estimates of the CAI components for cancellation, while the proposed iterative receiver further improves the performance by exploiting the non-circularity of the interference residual. The gain is smaller at low SNRs due to the dominance of the circular channel noise. As SNR increases, the performance gain by the proposed detector becomes larger since it benefits more from the exploiting the non-circularity of the interference.

The convergence behavior of the conventional ISIC and the proposed iterative schemes is examined in Fig. 3. Both converge at the 3 rd iteration, and further iterations do not yield noticeable performance improvement (the performance is almost identical at the $3 \mathrm{rd}$ and the 4th iterations). The conventional and the proposed schemes have the same performance at the first iteration since the pseudo-autocorrelation matrix $\tilde{\mathbf{C}}_{\mathbf{x x}}=\mathbf{0}$ at the initial stage as analyzed in Section IV. At the subsequent iterations, the interference terms become noncircular since $\tilde{\mathbf{C}}_{\mathbf{x x}}$ is non-vanishing due to a non-vanishing matrix $\tilde{\Lambda}_{n}$. Consequently, utilizing the non-circular nature of the interference yields significant performance gain.

\section{CONCLUSIONS}

A novel iterative detection scheme for MIMO-OFDM systems has been proposed in this paper. It has been shown that the conventional iterative solution is suboptimal and its performance can be improved by exploiting the non-circularity of the interference canceled signal at both the input and output of the nulling filter. The proposed scheme is compared to the conventional V-BLAST and ISIC schemes and is shown to achieve superior performance.

\section{REFERENCES}

[1] E. Telatar. "Capacity of multi-antenna Gaussian channels". European Transactions on Telecommunications, vol. 10, no. 6, pp. 585-595, Nov. 1999.

[2] L. Cimini. "Analysis and simulation of a digital mobile channel using orthogonal frequency division multiplexing". IEEE Transactions on Communications, vol. 33, no. 7, pp. 665-675, July 1985.

[3] J. Bingham. "Multicarrier modulation for data transmission: an idea whose time has come". IEEE Communications Magazine, vol. 28, no. 5, pp. 5-14, May 1990.

[4] S. Haykin, M. Sellathurai, Y. De Jong, T. Willink. "Turbo-MIMO for wireless communications". IEEE Communications Magazine, vo. 42, no. 10, pp. 48-53, Oct. 2004.

[5] X. Wautelet, A. Dejonghe, L. Vandendorpe. "MMSE-based fractional turbo receiver for space-time BICM over frequency-selective MIMO fading channels". IEEE Transactions on Signal Processing, vo. 52, no. 6, pp. 1804-1809, June 2004.

[6] G. Foschini. "Layered space-time architecture for wireless communication in a fading environment when using multi-element antennas". Bell Labs Technical Journal, pp. 41-59, 1996.

[7] G. Golden, G. Foschini, R. Valenzuela, P. Wolniansky. "Detection algorithm and initial laboratory results using the V-BLAST space-time communication architecture". IEE Electronic Letters, vol. 35, no. 1, pp. 14-16, January 1999.

[8] T. Ito, X. Wang, Y. Kakura, M. Madihian, A. Ushirokawa. "Performance comparison of MF and MMSE combined iterative soft interference canceller and V-BLAST technique in MIMO/OFDM systems". Proc. IEEE Vehicular Technology Conference, vol. 1, pp. 488-492, 2003.

[9] S. Ahmed, T. Ratnarajah, M. Sellathurai, C. Cowan. "Iterative receiver for MIMO-OFDM and their convergence behavior". IEEE Transactions on Vehicular Technology, vol. 58, no. 1, pp. 461-468, January 2009.

[10] J. Ylioinas, M. Juntti. "Iterative joint detection, decoding and channel estimation in turbo-coded MIMO-OFDM". IEEE Transactions on Vehicular Technology, vol. 58, no. 4, pp. 1784-1796, May 2009.

[11] C. Esli, M. Koca, H. Delic. "Iterative joint tone-interference cancellation and decoding for MIMO-OFDM". IEEE Transactions on Vechicular Technology, vol. 57, no. 5, pp. 2843-2855, September 2008.

[12] V. Poor, S. Verdu. "Probability of error in MMSE multiuser detection". IEEE Transactions on Communications, vol. 43, pp. 858-971, May 1997.

[13] S. Kay. Fundamentals of statistical signal processing, Prentice Hall, NJ, 1998.

[14] B. O'Hara, A. Petrick. IEEE 802.11 Handbook: A Desinger's Companion. 2nd Edition, IEEE, 2005. 